\title{
Study on the Interaction of Serum Albumin and Micromolecule Compounds
}

\author{
RUI HUANG ${ }^{\mathrm{a}, \mathrm{b}}, \mathrm{ZHI}^{-N I N G} \mathrm{XIA}^{* \mathrm{a}, \mathrm{c}}$, MIN YI $^{\mathrm{b}}$, AND BO-LIN LIU $^{\mathrm{c}}$ \\ ${ }^{\mathrm{a}}$ College of Bioengineering, Chongqing University \\ Chongqing 400044, P.R. China \\ ${ }^{\mathrm{b}}$ College of Criminal Investigation Law \\ Southwest University of Political Science and Law \\ Chongqing 401120, P.R. China \\ ${ }^{\mathrm{c}}$ College of Chemistry and Chemical Engineering \\ Chongqing University, Chongqing 400044, P.R. China \\ chem_lab@yahoo.cn
}

Received 9 July 2011; Accepted 20 September 2011

\begin{abstract}
Fluorescence spectrometry is a classical and conventional technique widely used in interaction investigation, which takes serum albumin as the fluorescence source while micro-molecule compound as the quencher. With analyzing the properties of serum albumin and its amino acids, deficiencies in the conventional fluorescence spectrometry became obvious: information expressed by the conventional technique was not sufficient to represent the entire interaction. In consideration of that, a new method was proposed to improve the conventional one. Intercompared the experimental results of those two methods, defects of the conventional fluorescence spectrometry were noticeable. In addition, molecular docking simulation and binding force investigation also manifested the same as above.
\end{abstract}

Keywords: Serum Albumin; Interaction Analysis; Fluorescence Spectrometry; Binding constant.

\section{Introduction}

The fluorescence spectrometry for interaction analysis between serum albumin (SA) and micromolecule has been focused and extensively studied ${ }^{[1-7]}$. For obtaining the binding constant $\mathrm{K}_{\mathrm{B}}$, the ultraviolet light at $280 \mathrm{~nm}$ is usually used as excitation beam, and the maximum emission wavelength $\left(\lambda_{\mathrm{em}}\right)$ is usually at $340 \mathrm{~nm}$. Fluorescence intensity of SA will be quenched disciplinarily with adding ligands gradually. And then, the varieties at $340 \mathrm{~nm}$ are considered as the changes of SA property in the interaction process to caculate $K_{B}$. 
Serum albumin is composed of twenty kinds of amino acids, but only three are aromatic amino acids, i.e. tryptophan (Trp), tyrosine (Tyr), and phenylalanine (Phe), which can emit fluorescence. Their $\lambda_{\mathrm{em}}$ are respectively 348,303 , and $282 \mathrm{~nm}$. It is clear that $\lambda_{\mathrm{em}}$ of SA at $340 \mathrm{~nm}$ is the contribution from only Trp residues. But, only Trp is able to participate in the interaction? Hundreds of the other residues are only bystanders. Absolutely not. Just because they are not fluorescent or weaker fluorescence producers, their interaction information cannot be expressed in the fluorescence spectrum of SA. In brief, it is not appropriate to take the spectrum of Trp residues to evaluate the property changes of SA, even though it has been popularly used for long.

In this paper, the biomacromolecules of human serum albumin (HSA) and bovine serum albumin (BSA) were selected as experimental subjects. The interactions between HSA/BSA and several micro-molecule ligands were investigated in detail.

\section{Experimental}

Human serum albumin (HSA, Genview) and bovine serum albumin (BSA, Genview) were directly dissolved in double distilled water to prepare their stock solutions $\left(1.0 \times 10^{-3} \mathrm{~mol} \cdot \mathrm{L}^{-1}\right)$. Sodium Dodecyl Benzene Sulfonate (SDBS), Trihexyphenidyl (THP), Primaquine (PQ), Citalopram (CIT), Ephedrine (EP), Triton X-100 (TX), Sibutramine (SH), and Florfenicol (FL), the purities of the 8 pharmacies were all raw medicine or biochemical regent and the purity was not less than $98 \%$. The $\mathrm{pH} 7.0$ buffer containing $0.02 \mathrm{~mol} \cdot \mathrm{L}^{-1}$ phosphate and $0.10 \mathrm{~mol} \cdot \mathrm{L}^{-1} \mathrm{NaCl}$ was prepared. Analytical grade chemicals and double distilled water were used throughout the whole experiments. All fluorescence spectrometric experiments were carried out on a RF-5301PC spectrofluorimeter (Shimadzu, Japan) equipped with $1.0 \mathrm{~cm}$ quartz cells.

\section{Methodology}

Molecular docking simulation: Molecular docking was performed using ICM 3.3 software. The ligands were constructed in the HyperChem 7.0, while the structure of the HSA was downloaded from the RCSB Protein Data Bank (PDB; http://www.rcsb.org/pdb/ home/home.do). Both the ligands and HSA were pre-optimized using MM+ and AMBER force field, respectively.

Fluorescence spectrometry: A constant concentration of detected molecule was kept in several volumetric flasks of $10.0 \mathrm{~mL}$. Different volumes of quencher molecule and $2.0 \mathrm{~mL}$ buffer solution were added, and then diluted to $10.0 \mathrm{ml}$ with double distilled water.

Binding force patterns investigation: Fluorescence spectrometric experiments were operated under $10^{\circ} \mathrm{C}$ and $25^{\circ} \mathrm{C}$ respectively. Through the different $\mathrm{K}_{\mathrm{B}}$ values from different temperature environment, the thermodynamic information and binding force patterns could be attained.

\section{Results and Discussion}

\section{Molecular docking simulation}

HSA is a globular protein consists of 585 amino acids and comprises three homologous domains (denoted I, II, and III): I comprises residues 1-195, II residues 196-383 and III residues 384-585, with two subdomains A and B each, which possess common structural motifs. The principal regions of ligand binding to HSA are located in hydrophobic cavities in subdomains IIA and IIIA, and one tryptophan residue (Trp-214) of HSA is in subdomain IIA ${ }^{8}$. There is a hydrophobic cavity present in subdomain IIA can bind to many drugs. 
ICM technique proposed by Totrov ${ }^{9}$ was adopted to achieve the flexible docking of HSA and micromolecules. ICM is a fully flexible docking technique based on the MonteCarlo method. It estimates the docking effect by calculating the electrostatic, stereo, and hydrogen binding effect, also the conformational free energy. Docking of drug and biomacromolecule is commonly operated on defined active site, like protein pockets, cracks or cavities.

Taking SDBS, THP, PQ, and CIT binding with the subdomain IIA hydrophobic cavity in HSA respectively, SDBS, and THP were not suited within that cavity for their large bulk. The optimized structures of the compounds HSA-PQ and HSA-CIT were shown in Figure 1. It was obvious in Figure 1 (a) that PQ was adjacent to hydrophobic residues, such as Leu (238), Tyr (150), and Ala (291), and there were some hydrogen bonds between PQ and residues such as Arg (257) and Lys (195). Similarly in the structure of HSA-CIT, many kinds of residues in IIA hydrophobic cavity participated in the interaction, some residues by the hydrophobic force such as Leu (238), Trp (214), Ala (291), and Glu (292) and the other by hydrogen bond such as Ser (192), Lys (195), and Lys (199). In addition, the lowest binding energy of HSA-PQ and HSA-CIT were respectively $-42.54 \mathrm{kcal} / \mathrm{mol}$ and $-45.04 \mathrm{kcal} / \mathrm{mol}$. It was indicated that the binding abilities of the two micromolecules binding with HSA were almost equivalent.

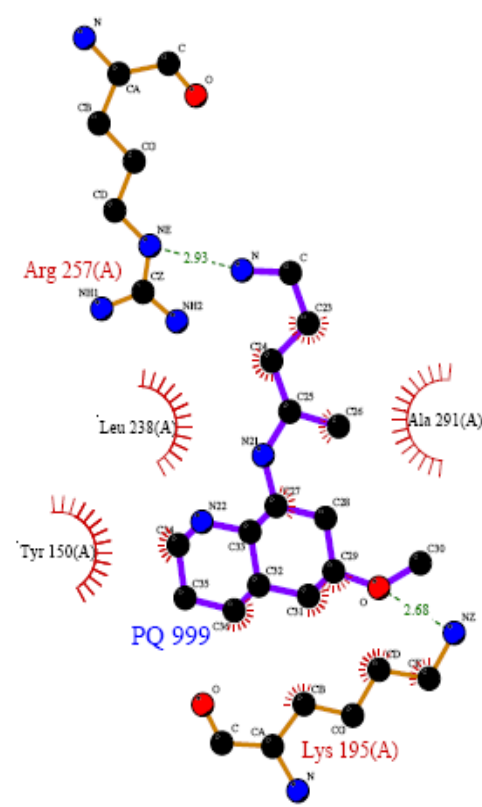

(a)

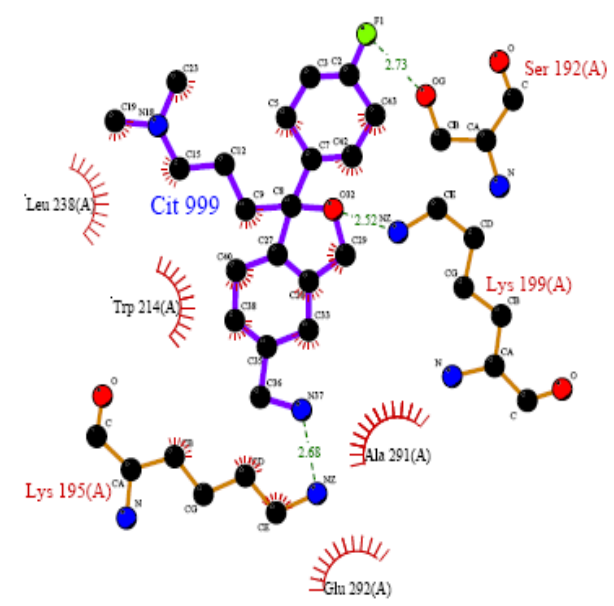

(b)

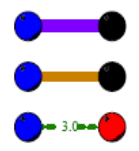

Ligand bond

Non-ligand bond

Hydrogen bond and its length

His 53 Non-ligand residues involved in hydrophobic

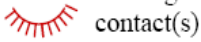

Corresponding atoms involved in hydrophobic contact(s)

Figure 1. The optimized structures of the compounds HSA-PQ (a) and HSA-CIT (b). 


\section{Fluorescence spectrometry}

\section{The conventional method}

In conventional fluorescence spectrometry (CFS), serum albumin is the detected object to supply fluorescence. Analyzing the various intensities at maximum emission wavelength under different concentrations of quencher, the binding constant $\mathrm{K}_{\mathrm{B}}$ can be calculated following the Stern-Volmer equation ${ }^{10}$ :

$$
\mathrm{F}_{0} / \mathrm{F}=1+\mathrm{K}_{\mathrm{sv}}[\mathrm{Q}]=1+\mathrm{K}_{\mathrm{q}} \tau_{0}[\mathrm{Q}]
$$

Where $\mathrm{F}$ and $\mathrm{F}_{0}$ are the fluorescence intensities with and without quencher, respectively. $\mathrm{K}_{\mathrm{q}}$ is the quenching rate constant of the biomolecule, $\mathrm{K}_{\mathrm{sv}}$ the Stern-Volmer quenching constant, $\tau_{0}$ the average lifetime of the biomolecule without quencher, about $10^{-8} \mathrm{~s}^{11}$ and [Q] is the concentration of quencher. The maximum scatter collision quenching constant $\mathrm{K}_{\mathrm{q}}$ of various quenchers with the biopolymer ${ }^{12}$ is about $2.0 \times 10^{10}(\mathrm{~mol} \cdot \mathrm{L})^{-1} \cdot \mathrm{s}^{-1}$. When the rate constant of the protein quenching procedure is greater than the maximum, it implies a static quenching process which originates from the formation of a complex. The static quenching equation can be expressed as ${ }^{13}$ :

$$
\lg \left[\left(\mathrm{F}_{0}-\mathrm{F}\right) / \mathrm{F}\right]=\operatorname{nlg}[\mathrm{Q}]+\lg \mathrm{K}_{\mathrm{b}}
$$

The four interaction systems, HSA-SDBS, HSA-THP, HSA-PQ, and HSA-CIT, were studied by CFS. It was observed in the spectra that with the addition of drugs, the fluorescence intensity of HSA decreased and the $\lambda_{\mathrm{em}}$ shifted to the shorter wavelength. Through Eq.(1), $\mathrm{K}_{\mathrm{q}}$ values of the four systems were calculated and all of them were greater than the maximum. According to Eq. $(2), \mathrm{K}_{\mathrm{HSA}-\mathrm{SDBS}}=6.74 \times 10^{2} \mathrm{~L} \cdot \mathrm{mol}^{-1}, \mathrm{~K}_{\mathrm{HSA}-\mathrm{THP}}=4.15 \times 10^{2} \mathrm{~L} \cdot \mathrm{mol}^{-1}$, $\mathrm{K}_{\mathrm{HSA}-\mathrm{PQ}}=5.01 \times 10^{3} \mathrm{~L} \cdot \mathrm{mol}^{-1}$ and $\mathrm{K}_{\mathrm{HSA}-\mathrm{CIT}}=3.50 \mathrm{~L} \cdot \mathrm{mol}^{-1}$. Compared with HSA-PQ, the bonding strength of HSA-CIT was much weaker. That was inconsistent with the result of macular modeling study which revealed the comparative binding intensities of the two interaction systems.

\section{The modified method}

Among the 585 amino acid residues in HSA, there is only one Trp residue located ${ }^{[14]}$. It is hard to confirm that the interaction only takes place at Trp residues or affects its microenvironment. Therefore, the inaccuracy of CFS is derived from taking the partial information as the entire. In the other words, accurate binding constant $\mathrm{K}_{\mathrm{B}}$ can be obtained only when fluorescence information express the total information of the detected molecule. Fortunately, for a fluorescent micromolecule, its fluorescence can obviously represent the properties of the entire molecule. The method, taking the micro-molecule ligand as detected analyte and investigating the effect induced by serum albumin, is called modified fluorescence spectrometry (MFS) in this paper. For a same interaction system, two $\mathrm{K}_{\mathrm{B}}$ values can be attained respectively by CFS and MFS. To some extent, the one-sidedness of CFS can be proved when the $\mathrm{K}_{\mathrm{B}}$ from CFS is much smaller than the $\mathrm{K}_{\mathrm{B}}$ from MFS.

In addition, it is still an obstacle to overcome if the fluorescence of the two fluorescent molecules in a same interaction system interfering each other terribly, because of the strong fluorescence of Trp in HSA. Aiming at the overlapping fluorescence spectra, the fluorescence background subtracting method (FBS) was proposed. In this method, the disturbed spectrum of the detected object $\left(\mathrm{O}_{\mathrm{b}}\right)$ is considered as its apparent spectrum and the interfering spectrum of the quencher $\left(Q_{u}\right)$ as the background interference. A series of $Q_{u}$ 
solutions as reference solutions were prepared, whose concentrations are accordant with those in apparent spectrum. Under the same detection conditions, spectra of reference solutions are recorded as the background interference spectra. Subtracting the background from the apparent spectra, the actual spectra of $\mathrm{O}_{b}$ can be obtained. These heights of peaks in treated spectra are to be used for calculation of $\mathrm{K}_{\mathrm{B}}$.

Taking SDBS, THP, PQ, and CIT as fluorescence sources as MFS: (a) as shown in Figure 2, addition of HSA caused a regular reduction in the fluorescence emission intensity of SDBS, THP, and PQ and an isoemissive point appeared; (b) due to the fluorescence contribution of HSA at $299 \mathrm{~nm}$, the spectrum of CIT distorted. Dealt with FBS, it could be observed from the actual spectrum that the fluorescence emission intensity of CIT regularly decreased between 200-400 $\mathrm{nm}$ (shown as Figure 3). Binding constants of the four systems

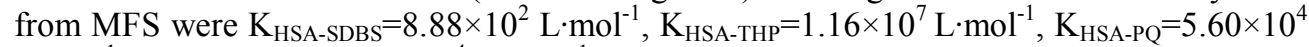
$\mathrm{L} \cdot \mathrm{mol}^{-1}$ and $\mathrm{K}_{\mathrm{HSA}-\mathrm{CIT}}=5.86 \times 10^{4} \mathrm{~L} \cdot \mathrm{mol}^{-1}$, and the $\mathrm{K}_{\mathrm{B}}$ of HSA-PQ and HSA-CIT were accordant with the result of molecular modeling study in 3.1 .

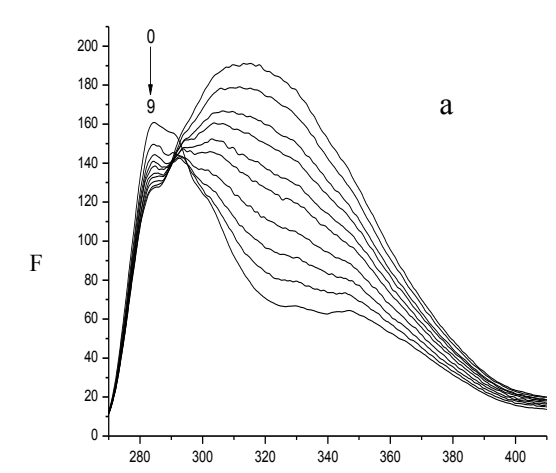

Wavelength/nm

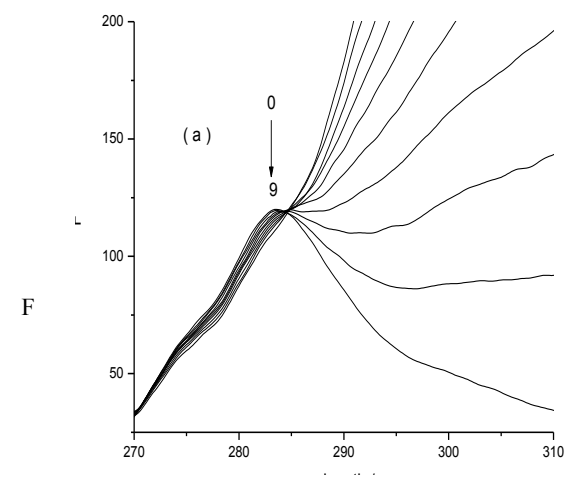

Wavelength/nm

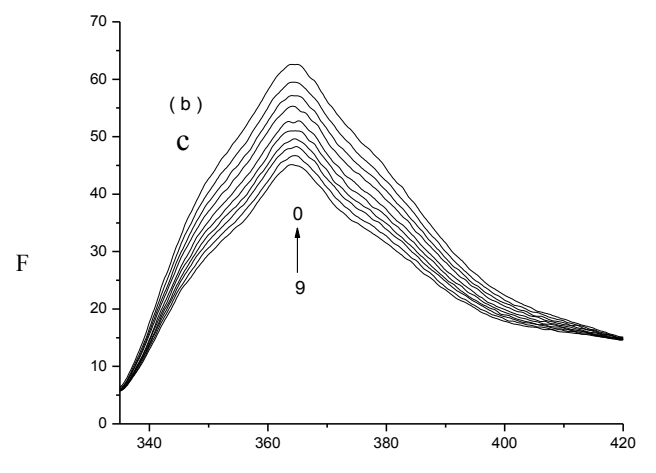

Wavelength/nm

Figure 2. The fluorescence spectra of SDBS (a), THP (b), and PQ (c) quenched by HAS (a) $[\mathrm{SDBS}]=3.47 \times 10^{-5} \mathrm{~mol} / \mathrm{L}$, [HSA] from 0 to 9 : $0,0.17,0.33,0.50,0.66,0.82,0.98,1.14,1.30$, $1.46 \times 10^{-6} \mathrm{~mol} / \mathrm{L}$, (b) $[\mathrm{THP}]=1.33 \times 10^{-4} \mathrm{~mol} / \mathrm{L}$, [HSA] from 0 to $9: 0,0.50,0.99,1.49,1.97$, $2.46,2.94,3.42,3.90,4.37 \times 10^{-6} \mathrm{~mol} / \mathrm{L}$, (c) $[\mathrm{PQ}]=1.00 \times 10^{-4} \mathrm{~mol} / \mathrm{L}$, [HSA] from 0 to 9 : $0,0.17,0.33,0.50,0.66,0.82,0.98,1.14,1.30,1.46^{*} 10^{-6} \mathrm{~mol} / \mathrm{L}$. 


\section{Comparing CFS with MFS and their available application}

Compared the results of the four systems as in Table 1, it was obvious that binding constants from MFS were much greater than those from CBS except HSA-SDBS system. (1) HSASDBS system: The two $\mathrm{K}_{\mathrm{B}}$ values from CFS and MFS were comparative and were both weak.
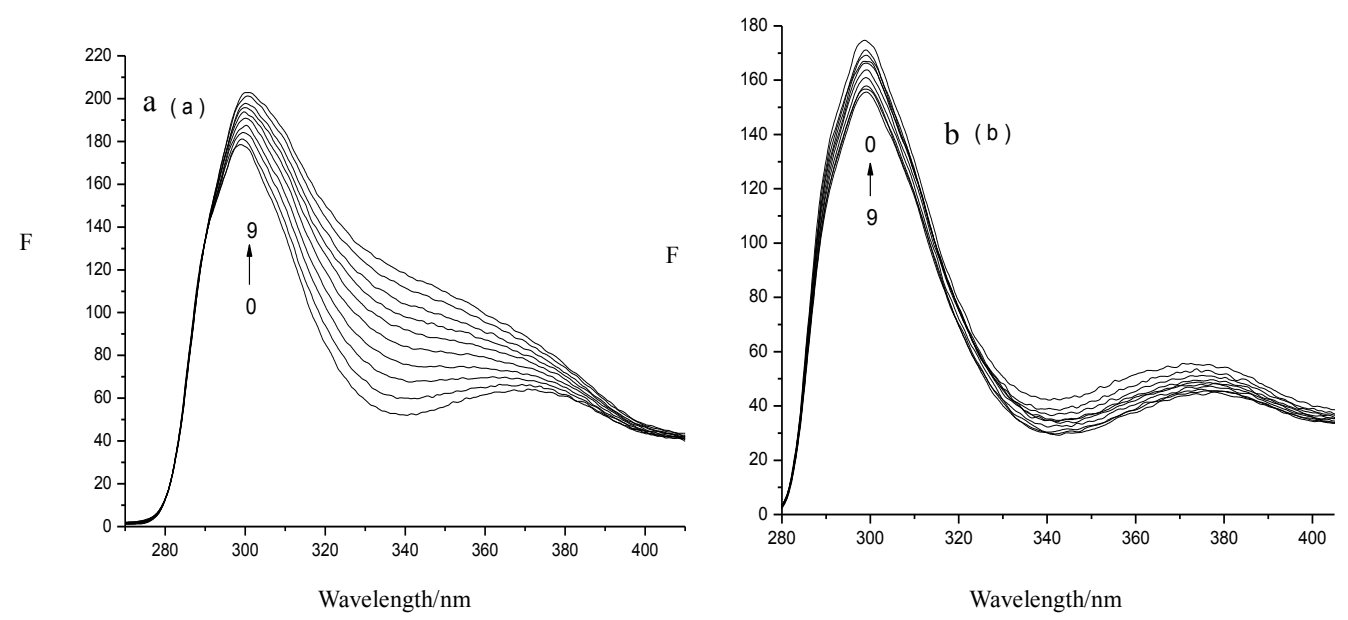

Figure 3. The interfered fluorescence spectrum (a) and the actual fluorescence spectrum (b) of CIT quenched by HAS [CIT] $=4.0 \times 10^{-5} \mathrm{~mol} / \mathrm{L}$, [HSA] from 0 to $9: 0,0.17,0.33,0.50,0.66$, $0.82,0.98,1.14,1.30,1.46 \times 10^{-6} \mathrm{~mol} / \mathrm{L}$.

Considering the result of molecular docking simulation, the size of SDBS was too big to insert the IIA hydrophobic cavity. So the interaction might take place in other domains or subdomains and the changes of fluorescence might be induced by the alteration of HSA structure. (2) HSA-THP and HSA -PQ systems: The $\mathrm{K}_{\mathrm{B}}$ from MFS was greater than the value from CFS. The spectrum of MFS reflected more information contributed by more other amino acids which could not be expressed in the spectrum of CFS. (3) HSA-CIT system: The binding force reflected in CFS was so weak but the result from MFS was completely reverse. It demonstrated that the interaction affected the Trp residue little and its main effect occurred on other kinds of residues whose information was not shown in CFS. From the above experiments, the binding constant of HSA-PQ by MFS was close to that of HSA-CIT by MFS and FBS, which matched well with the result of molecular docking study.

Based on aforementioned theoretical derivation and experienns, it can be deduced that fluorescence spectrometry is suitable for three typesof the SA-micromolecule interaction systems (shows in Table 1). Type I : the binding force between SA and micromolecule is weak and the interaction information can be centrally reflected on Trp residue. For this kind of system, the effects on other residues expect Trp residue can be ignored and both CFS and MFS are applicable. Type II : other amino acids or groups may actively participate in the interaction, thus Trp residue cannot express the entire information. For this type, it is more reliable to use micro-molecule ligand as the fluorescence source. Type III: Trp residue cannot represent the whole information, and the spectrum of micromolecule is disturbed by the strong fluorescence of SA. Combination of MFS and FBS is available for such systems. 
Table 1. The comparison of $\mathrm{K}_{\mathrm{B}}\left(\mathrm{L} \cdot \mathrm{mol}^{-1}\right)$ of HSA-micromolecules from CFS and MFS.

\begin{tabular}{ccccc}
\hline & Type I & \multicolumn{2}{c}{ Type II } & Type III \\
\hline & HSA-SDBS & HSA-THP & HSA-PQ & HSA-CIT \\
$\mathrm{K}_{\mathrm{B}}$ from CFS & $6.74 \times 10^{2}$ & $4.15 \times 10^{2}$ & $5.01 \times 10^{3}$ & 3.50 \\
$\mathrm{~K}_{\mathrm{B}}$ from MFS & $8.88 \times 10^{2}$ & $1.16 \times 10^{7}$ & $5.60 \times 10^{4}$ & $5.86 \times 10^{4}$ \\
\hline
\end{tabular}

\section{Binding force patterns investigation}

Hydrophobic binding force, hydrogen bonding force, Van Der Waals force and electrostatic binding force are the main binding forces between organic micromolecule and biomacromolecule ${ }^{15}$. According to abundant dada, Ross etc. ${ }^{16}$ summarized the thermodynamic rules to estimate the binding force between micromolecule and biomacromolecule. Comparing the relative enthalpy change $(\Delta \mathrm{H})$ and relative entropy $(\Delta \mathrm{S})$ change of pre-and post reaction, the main binding forms can be judged: $\Delta \mathrm{H}>0$ and $\Delta \mathrm{S}>0$, Hydrophobic binding force; $\Delta \mathrm{S}<0$ and $\Delta \mathrm{H}<0$, hydrogen bonding force and Van Der Waals force; $\Delta \mathrm{H}<0$ and $\Delta \mathrm{S}>0$, Electrostatic binding force. Free energy change $(\Delta \mathrm{G})$ can be calculated from $\Delta \mathrm{G}=-\mathrm{RT} \ln \mathrm{K}_{\mathrm{B}} \Delta \mathrm{H}$ can be got from $\mathrm{K}_{\mathrm{B}, 1}$ and $\mathrm{K}_{\mathrm{B}, 2}$ of different temperature $\left(10^{\circ} \mathrm{C}\right.$ and $\left.25^{\circ} \mathrm{C}\right), \Delta \mathrm{H}=\mathrm{RT}_{1} \mathrm{~T}_{2} \ln \left[\mathrm{K}_{\mathrm{B}, 2} / \mathrm{K}_{\mathrm{B}, 1}\left(\mathrm{~T}_{2}-\mathrm{T}_{1}\right)\right]$. Subsequently, $\Delta \mathrm{S}$ will be calculated by $\Delta \mathrm{G}$ and $\Delta \mathrm{H}$.

According to the above-mentioned theory, the main binding forms of the four systems were clear: (1) both by CFS and MFS, the main binding style of HSA-SDBS, HAS-PQ, and HSA-CIT was the same, hydrophobic binding force; (2) for HSA-THP system, it was hydrophobic binding force by CFS, but electrostatic binding force by MFS. The phenomena were accordant with the results in 3.1. Considering Figure 1, many active amino acids interacted with the micro-molecule ligand by hydrophobic bind, such as Leu (238), Tyr (150), and Ala (291) in HSA-PQ system and Leu (238), Trp (214), Ala (291), and Glu (292) in HSA-CIT system. All those residues were active participants of the interaction and the hydrophobic binding force was their main interaction mode. As known for HSA-SDBS, the binding constants from CFS and MFS were almost equivalent and both weak. It indicated there was a kind of weak binding force between HSA and SDBS, such as the hydrophobic. Conversely, $\mathrm{K}_{\mathrm{B}}$ of HSA-THP from MFS was the greatest in Table 1, which demonstrated the bind between HSA and THP was strong, such as the electrostatic binding force. And for this kind of systems, it was not appropriate adopting CFS to investigate, because the interaction occurred not in subdomain IIA, where the only one Trp residue located.

However, why the fluorescence of Trp changed while SDBS and THP could not insert subdomain IIA. There might be two reasons: (1) The interaction induced the HSA structure changed and the microenvironment of Trp changed subsequently. (2) The emission wavelength of Phe and Tyr both could excite the fluorescence of Trp. The fluorescence changes of Trp were likely aroused by the property changes of Phe or Tyr.

\section{The interaction between BSA and micromolecules}

Because of its similar structure and property with HSA, BSA is also a focus SA in the interaction study. BSA molecule contains 582 amino acid residues, of which only two are tryptophan residues (Trp-134 and Trp-212) ${ }^{17}$. Based on previous work ${ }^{18,19}$, eight interaction systems viz. BSA-SDBS, BSA-THP, BSA-PQ, BSA-TX, BSA-FL, BSA-EP, BSA-CIT, and 
BSA-SH, were studied by CFS and MFS, and the interaction information was showed in Table 2.

\section{$B S A-S D B S$ system}

The spectra information and binding constants from CFS and MFS were well matched with each other. In both methods, the $\lambda_{\mathrm{em}}$ of detected molecule shifted to the shorter, which expressed a same interaction site or a same microenvironment.

Table 2. The interaction information of BSA - micromolecules from CFS and MFS.

\begin{tabular}{|c|c|c|c|c|c|c|c|c|c|}
\hline & $\begin{array}{l}\mathrm{A}- \\
3 \mathrm{~S}\end{array}$ & $\begin{array}{l}\text { BSA- } \\
\text { THP }\end{array}$ & BSA & BSA & $\begin{array}{c}\text { BSA- } \\
\text { EP }\end{array}$ & $\begin{array}{c}\text { BSA- } \\
\text { FL }\end{array}$ & $\begin{array}{c}\text { BSA- } \\
\text { SH }\end{array}$ & CIT & $\begin{array}{l}\text { BSA- } \\
\text { SDBS }\end{array}$ \\
\hline & $\lambda_{\mathrm{em}}$ & $\begin{array}{l}\text { blue } \\
\text { shift }\end{array}$ & blue & & $\begin{array}{l}\text { blue } \\
\text { shift }\end{array}$ & $\begin{array}{l}\text { blue } \\
\text { shift }\end{array}$ & $\begin{array}{l}\text { blue } \\
\text { shift }\end{array}$ & & \\
\hline & $\begin{array}{c}\mathrm{K}_{\mathrm{B}} / \\
\mathrm{L} \cdot \mathrm{mol}^{-1}\end{array}$ & $00 \times 10^{6}$ & $5.04 \times 10^{3}$ & $10 \times 10^{3}$ & $7.77 \times 10^{2}$ & $29 \times 10^{2}$ & $.84 \times 10^{4}$ & & \\
\hline \multirow[b]{2}{*}{ CFS } & $\lambda_{\mathrm{em}}$ & e shift & & red s & red shift & 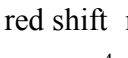 & red shift & ift & ed shi \\
\hline & $\begin{array}{c}\mathrm{K}_{\mathrm{B}} / \\
\mathrm{L} \cdot \mathrm{mol}^{-}\end{array}$ & $15 \times 10^{6}$ & $4.12 \times 10^{5}$ & $7.99 \times 10^{4}$ & $4.58 \times 10^{7}$ & $8.40 \times 10^{4}$ & $4.14 \times 10^{6}$ & $1.38 \times 10^{6}$ & $74 \times 10^{7}$ \\
\hline
\end{tabular}

Systems BSA-THP, BSA-PQ, BSA-TX, BSA-EP, BSA-FL and BSA-SH: In CFS spectra, the $\lambda_{\text {em }}$ of BSA-SH system shifted to red, and the others shifted to blue. But in MFS spectra, the $\lambda_{\text {em }}$ of every micromolecule shifted to the reverse direction of BSA in CFS. The red shift was ascribed to a lowering polarity in the protein environment around Trp residues, and the blue shift described an increasing polarity environment ${ }^{[20,21]}$. The contrary spectra information of CFS and MFS expressed property changes of different interaction sites or different microenvironment. Moreover, the binding constants from MFS were all greater than those from CFS. The two phenomena both illustrated the deficiencies of CFS.

BSA-CIT system: It was impossible to get available spectrum by CFS, because the weak and irregular fluorescence changes of BSA. The same phenomenon occurred in the system Trp-CIT by taking pure Trp residue solution as detected molecule as CFS. It could be deducted that CIT affected the Trp little and CFS was not available. Dealt by MFS and FBS, its $\mathrm{K}_{\mathrm{B}}$ was $2.74 \times 10^{7} \mathrm{~L} \cdot \mathrm{mol}^{-1}$ and the relative coefficient $\left(\mathrm{r}^{2}\right)$ of the curve was 0.9976 .

\section{Conclusion}

Through comparisons of the experiment results obtained from molecular docking simulation, fluorescence spectrometry and binding force pattern investigation, deficiency and inaccuracy of the conventional fluorescence spectrometry were revealed. Molecular docking technique was used for verifying the roles of amino acid residues in the interaction process. Choose HSA and micromolecules as fluorescent source respectively, significant different spectra information and binding constants were obtained, which well suggested Trp spectrum information can not estimate the whole interaction accurately. The results of thermodynamic study indicated the main binding force in the interaction system was hydrophobic interaction. In addition, the interaction results between BSA and eight micromolecules also confirmed the deficiency of the conventional fluorescence spectrometry.

Furthermore, two modified methods were proposed: MFS could supply more comprehensive interaction information, while FBS could optimize the lapping spectra. With their advantages, application of fluorescence spectrometry has been expanded. In other words, any interaction system containing one or more fluorescent molecular can be investigated by MFS or the combination of MFS and FBS. 


\section{Acknowledgment}

We gratefully acknowledge the financial support of the National Natural Science Foundation of China (No. 20805060), the Natural Science Foundation of Chongqing Science and Technology Commision, China (No.CSTC, 2008BB9266), the Science Foundation of Chongqing Municipal Commission of Education, China (No. KJ100102), and the Fundamental Research Funds for the Central Universities, China (No.CDJXS10221136).

\section{References}

1. Gelamo E L and Tabak M, Spectrochim Acta A, 2007, 56, 2255.

2. Caballero-Quintero A, Piñeyro-López A and Waksman N, Int J Pharm., 2001, 229, 23.

3. Gelamo E L, Silva C H T P, Imasato H and Tabak M, Biochim Biophys Acta, 2002, 1594, 84.

4. Gizzi P, Henry B, Rubini P, Giroux S and Wenger E, J Inorg Biochem., 2005, 99, 1182.

5. Bordbar A K and Asghar T K, Colloids Surf B, 2007, 55, 84.

6. Zhang Y Z, Xiang X, Mei P, Dai J, Zhang L L and Liu Y, Spectrochim Acta A, 2009, 72, 907.

7. Kathiravan A, Chandramohan M, Renganathan R and Sekar S, J Mol Struct., 2009, 919, 210.

8. Carter D C, He X M, Munson S H, Twigg P D, Gernert K M, Broom M B and Miller T Y, Science, 1989, 244, 1195.

9. Abagyan R A, Totrov M M and Kuznetsov D A, J Comput Chem., 1994, 15, 488.

10. Lakowicz J R, Principles of Fluorescence Spectroscopy ( $2^{\text {nd }}$ Edition), Plenum Press, New York, 1999, 237-265.

11. Lakowicz J R and Weber G, J Biochem., 1973, 12, 4161.

12. Ware W R, J Phys Chem., 1962, 66, 455.

13. Aveline B M, Hasan T and Redmond R W, J Photochem Photobiol B, 1995, 30, 161.

14. Sugio S, Kashima A, MochlzukI S, Noda M and Kobayashi K, J Protein Eng., 1999, 12, 439.

15. Gonzalez-Jzmenoz J, Jacquotte H and Cayre I, Chem Biol Interact., 1992, 84, 221.

16. Ross P D and Subramanian S, J Biochem., 1981, 20, 3096.

17. Moriyama Y, Ohta D, Hachiya K, Mitsui Y and Takeda K J, J Protein Chem., 1996, 15, 265.

18. Huang R, Xia Z N and Gong P, Spectrosc Spect Anal., 2008, 28, 161.

19. Tan T, Huang R and Xia Z N, Chinese J Anal Chem., 2007, 35, 1415.

20. Mallick A, Bera S C, Maiti S and Chattopadhyay N, Biophys Chem., 2004, 112, 9.

21. Lu Q, Gu J S, Yu H P, Liu C, Wang L and Zhou Y Y, Spectrochim Acta A, 2007, 68, 15. 


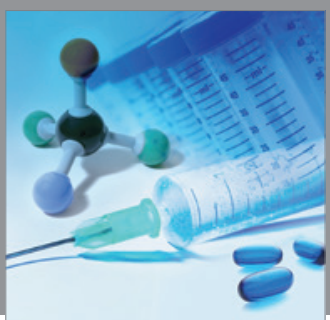

International Journal of

Medicinal Chemistry

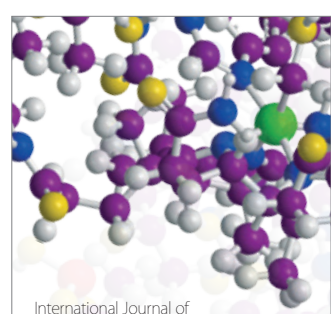

Carbohydrate Chemistry

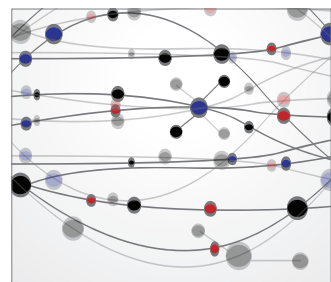

The Scientific World Journal
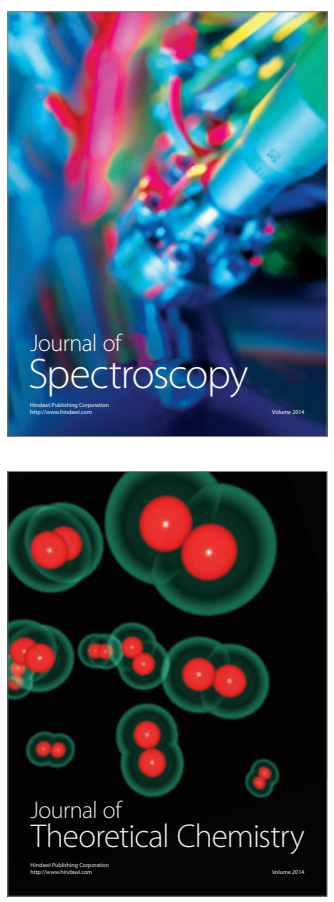
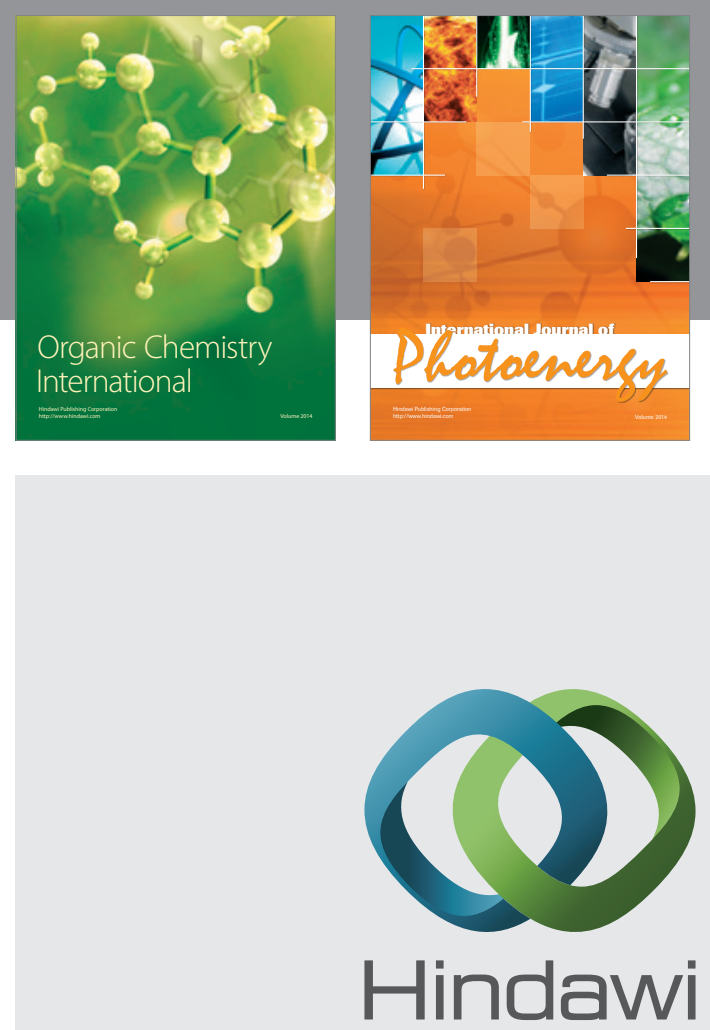

Submit your manuscripts at

http://www.hindawi.com
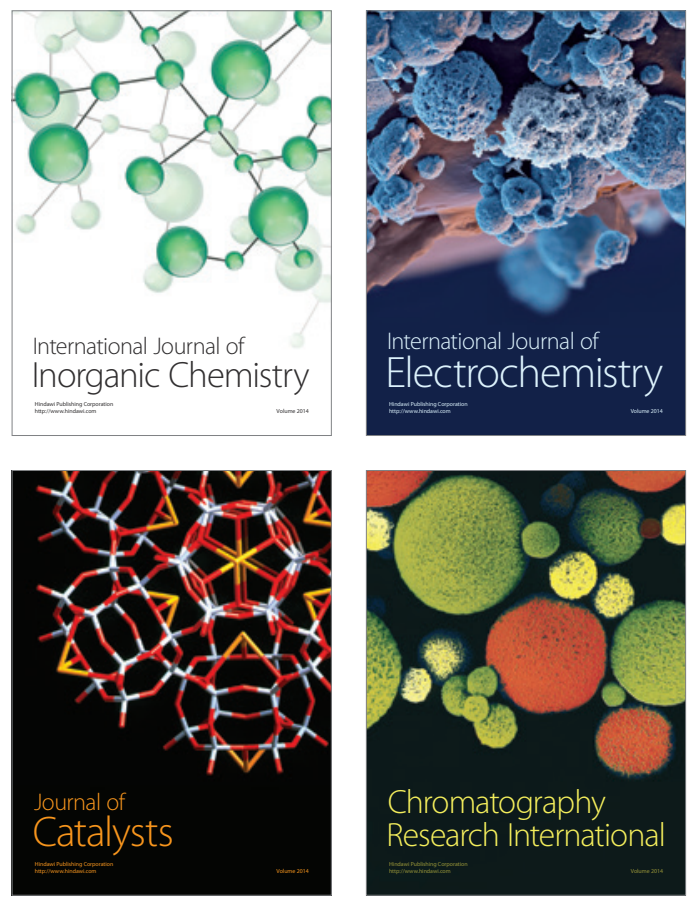
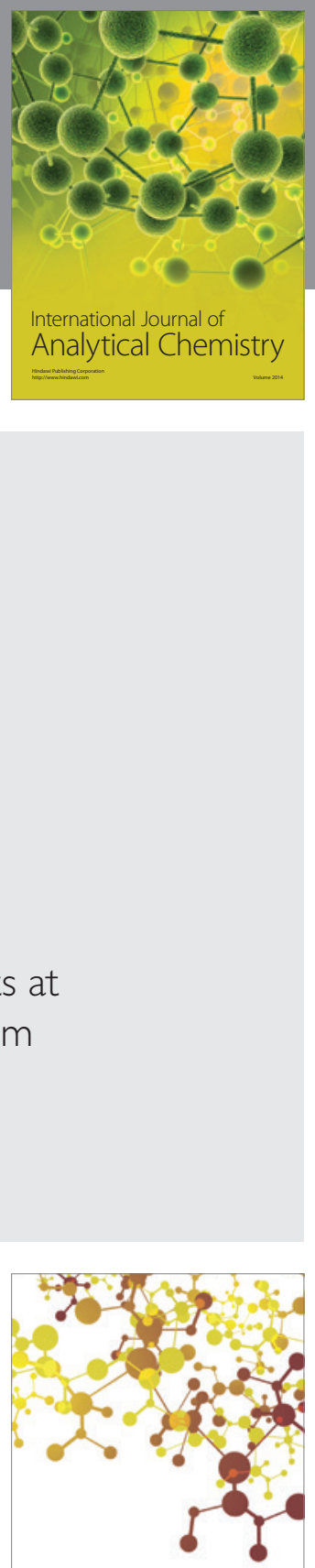

Journal of

Applied Chemistry
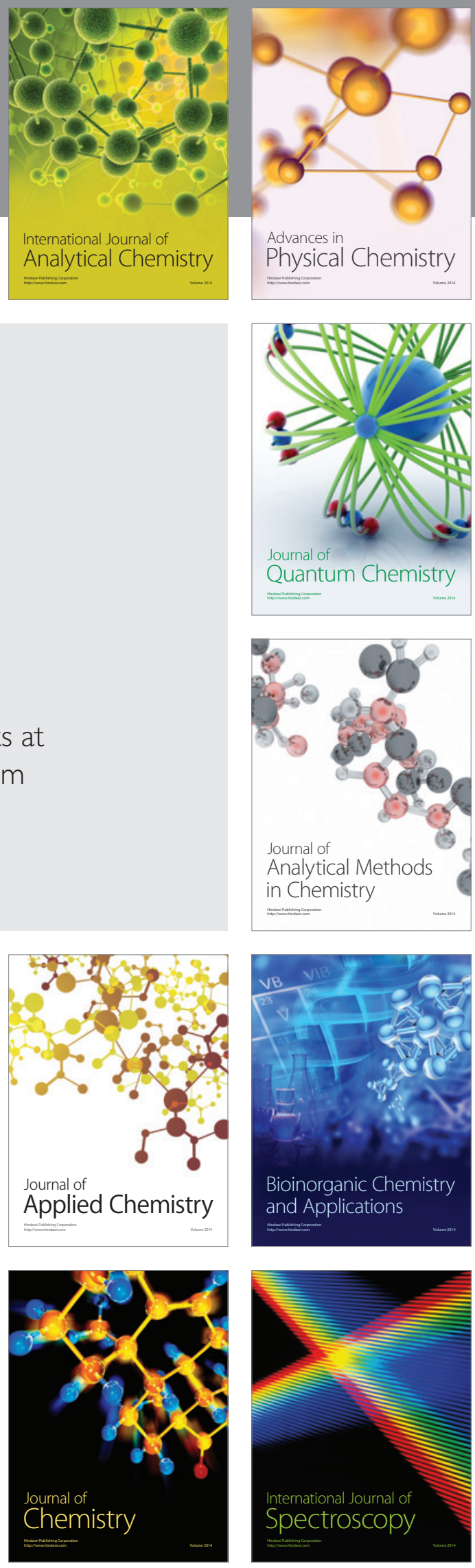